\title{
Expektoration von Embolisationsspiralen 15 Jahre nach Embolisation pulmonaler arteriovenöser Malformationen bei hereditärer hämorrhagischer Teleangiektasie (M. Osler-Weber-Rendu)*
}

\author{
Expectoration of Embolization Coils 15 Years after Embolization of Pulmonary Arteriovenous Malformations \\ in a Patient with Hereditary Hemorrhagic Telangiectasia
}

Autoren

Institute
D. Schwarzer ${ }^{1}$, I. Mäder ${ }^{1}$, A. Petrovitch ${ }^{2}$, J. Leonhardi ${ }^{3}$, R. Bonnet ${ }^{1,4}$

${ }^{1}$ Klinik für Pneumologie, Zentralklinik Bad Berka

${ }^{2}$ Klinik für interventionelle Radiologie, Zentralklinik Bad Berka

${ }^{3}$ Klinik für diagnostische Radiologie, Zentralklinik Bad Berka

${ }^{4}$ Loma Linda University, California eingereicht $\quad 6.11 .2013$ akzeptiert nach Revision 4.2.2014

Bibliografie

Dol http://dx.doi.org/

10.1055/s-0034-1365127

Online-Publikation: 10.3.2014

Pneumologie 2014; 68: 282-285

(c) Georg Thieme Verlag KG

Stuttgart · New York

ISSN 0934-8387

Korrespondenzadresse

Dr. med. Doina Schwarzer

Assistenzärztin

Klinik für Pneumologie

Zentralklinik Bad Berka

Robert-Koch-Allee 9

99437 Bad Berka

pne@zentralklinik.de

\section{Zusammenfassung}

$\nabla$

Bei der hereditären hämorrhagischen Teleangiektasie (HHT, Morbus Osler-Weber-Rendu) kann es zur Ausbildung von pulmonalen arteriovenösen Malformationen (pAVM) kommen. Zur Vermeidung von Komplikationen und zum Verschluss relevanter AV-Shunts sollte eine Embolisation der Malformationen vorgenommen werden. Im vorliegenden Fallbeispiel kam es zum Abhusten von Embolisationsspiralen 15 Jahre nach Intervention. Bei Hämoptysen nach Interventionen mit Embolisationsspiralen sollte auch Jahre nach deren Implantation neben dem Verdacht auf neue AV-Malformationen an durchwanderte Spiralen gedacht und eine entsprechende Diagnostik durch Einsatz von Thorax-CT mit Kontrastmittel und Bronchoskopie durchgeführt werden.

\section{Einleitung}

Bei der hereditären hämorrhagischen Teleangiektasie (HHT, M. Osler-Weber-Rendu) kommt es zur Ausbildung von mukokutanen arteriovenösen Malformationen. Sie ist eine seltene Erkrankung mit einer Inzidenz von ca. 1:10000 und wird autosomal-dominant vererbt.

Die Erkrankung manifestiert sich bei $90 \%$ der Patienten durch rezidivierende Epistaxis [1]. Es zeigen sich Teleangiektasien vor allem an den Fingern und im Gesicht sowie im weiteren Verlauf AV-Malformationen innerer Organe. Besonders befallen sind Lunge, Gastrointestinaltrakt, Leber und Gehirn.

Speziell die Lunge ist zwischen 15 und 50\% befallen [2-4]. Meist sind die Unterlappen betroffen [5]. Hämoptysen und Zyanose sind die Hauptsymptome. Bei schweren Verläufen kann es auch zur Ausbildung einer pulmonalen Hypertonie, zu schwerer Hämoptoe oder zur Ausbildung eines Hämatothorax kommen [6]. Durch die Shuntverbindung können sich ischämische Insulte oder

\section{Abstract \\ $\nabla$}

Hereditary hemorrhagic telangiectasia can manifest itself with pulmonary arteriovenous malformations (pavm). A transcatheter coil embolization should be made to avoid complications and to close off relevant arteriovenous shunts. We report on a patient with expectoration of embolization coils 15 years after embolotherapy. In case of hemoptysis following embolotherapy with coils, even years after their placement one should consider coil migration into the pulmonary system, besides newly formed pavms, in the differential diagnosis and initiate contrast-CT of the thorax and bronchoscopy.

septische Embolien mit intrazerebralen Abszessen bilden. Zur Prävention eines fulminanten Verlaufs empfiehlt sich die frühe interventionelle Ausschaltung des AV-Shunts durch Coiling. Im ausgesuchten Fallbeispiel zeigt sich eine Durchwanderung des Embolisationsmaterials mit Anschluss an das Bronchialsystem 15 Jahre postinterventionell.

\footnotetext{
* Anmerkung: Der Fallberichtet handelt von einem Patienten, welcher in der Klinik für Pneumologie in Bad Berka behandelt wurde. Die Autorin ist Assistenzärztin in dieser Klinik. Die Arbeit erfolgte unter Supervision und Korrektur durch Frau Dr. Mäder und unter der Leitung von Herrn Prof. Dr. Bonnet. Mitbeteiligt waren Herr Dr. Petrovitch, welcher die Intervention mit Verklebung der AVFisteln durchführte und am Manuskript mitarbeitete, sowie Herr Dr. Leonhardi, welcher Hilfestellung bei der Befundung der CT-Bilder gab und freundlicherweise die CT-Befunde zur Verfügung stellt und am Manuskript mitarbeitete.
} 


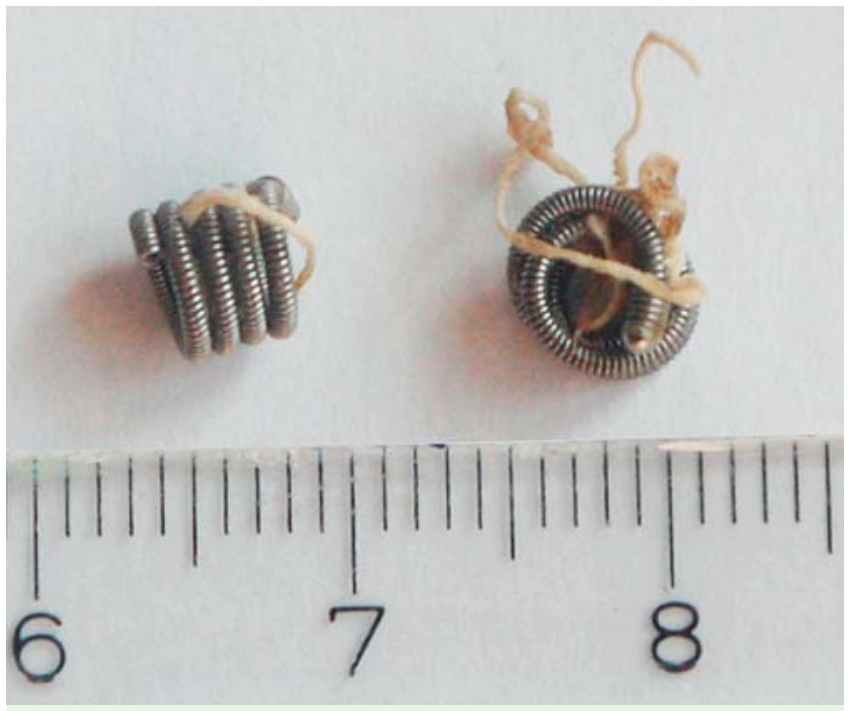

Abb.1 Abgehustete und entfernte Embolisationsspiralen.

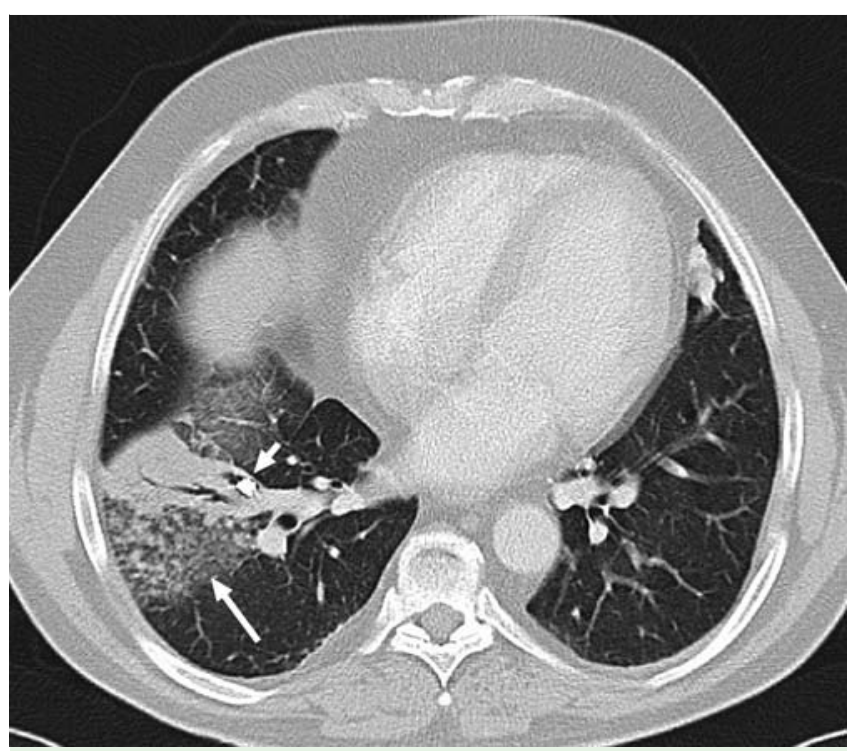

Abb.2 Intrapulmonale Einblutung (langer Pfeil), Embolisationsspirale (kurzer Pfeil).

\section{Kasuistik \\ $\nabla$}

Wir berichten über einen 54-jährigen Patienten, der sich mit seit zwei Wochen bestehenden Halsschmerzen, Husten und Auswurf sowie seit mehreren Wochen bestehender Epistaxis vorstellte. Am Aufnahmetag sowie zwei Tage zuvor hatte der Patient jeweils Embolisationsspiralen unter Hämoptysen abgehustet ( $\mathbf{A b b}$. 1). In der weiteren Vorgeschichte besteht eine bekannte hereditäre hämorrhagische Teleangiektasie mit multiplen arteriovenösen Malformationen. Hier wurden bereits 1992, 1997 und 1998 Embolisationen mehrerer pumonaler AV-Shunts beidseits durchgeführt. Des Weiteren besteht ein Zustand nach Tuberkulose 1972, zweifachen Pneumothoraces 1979 und einer zerebralen Ischämie mit sekundärem zerebralen Anfallsleiden sowie einer ShuntOperation 1992. Eine mikrozytäre hypochrome Anämie wurde bis 2010 medikamentös behandelt.

Der körperliche Untersuchungsbefund war ohne Auffälligkeiten. Laborchemisch fielen deutliche Entzündungszeichen (CrP 206,7 $\mathrm{mg} / \mathrm{l}$ ) und eine Anämie (Hb 5,2 mmol/l, Hkt 0,30) auf.

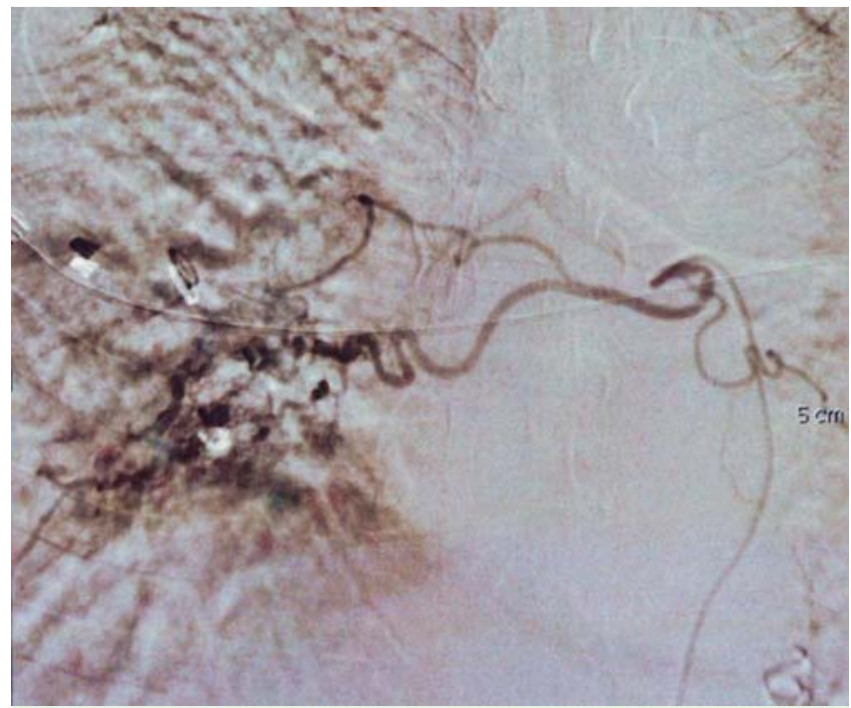

Abb.3 Unterlappensegmentarterie rechts.

Blutgasanalytisch wurde eine mittelgradige Hypoxämie mit akuter respiratorischer Alkalose $\left(\mathrm{pH} 7,49, \mathrm{pO}_{2}\right.$ 55,2, $\mathrm{pCO}_{2} 34,1$, $\left.\mathrm{CHCO}_{3-}(\mathrm{P}, \mathrm{st})_{\mathrm{c}} 26,7 \mathrm{mmol} / \mathrm{l}\right)$ und unter $100 \%$ iger $\mathrm{O}_{2}$-Atmung eine adäquate Oxygenierung ohne relevanten Shunt $\left(\mathrm{pH} 7,45, \mathrm{pO}_{2}\right.$ 399, $\mathrm{pCO}_{2} 36,8, \mathrm{CHCO}_{3-}$ (P,st) $\left.25,8 \mathrm{mmol} / \mathrm{l}, \mathrm{SBE}_{\mathrm{c}} 1,4 \mathrm{mmol} / \mathrm{l}\right)$ festgestellt.

Lungenfunktionell waren eine leichtgradige Restriktion und eine mittelgradig eingeschränkte Diffusionskapazität nachweisbar. In der Computertomografie des Thorax zeigte sich eine regelrechte Lage der Coils in den Segmenten 2, 4 und 5 rechts sowie in Segment 9 links, unverändert zur Voraufnahme von 08/2004. Im Gegensatz dazu waren die hintereinander eingebrachten Embolisationsspiralen in der Segmentarterie in S 8 rechts nicht mehr abzugrenzen. Neu zeigte sich eine größere, segmentale Konsolidierung im Segment 8 rechts mit angrenzender Milchglastrübung im Sinne einer intrapulmonalen Einblutung ( $\bullet$ Abb.2).

In der nachfolgenden Angiografie der Bronchialarterien stellten sich im Abstromgebiet der Unterlappenarterie rechts um die früher gecoilten AV-Shunts multiple Gefäßtransformationen mit einer Rarefizierung der Strombahn dar sowie insgesamt drei kleine neue AV-Shunts. Die originären Gefäße waren größtenteils um die Coils herum obliteriert und durch zahlreiche, kleinste Kapillaren als Kollateralen ersetzt $(\bullet$ Abb. 3).

Bronchoskopisch war eine Blutspur im rechten Unterlappen auf Subsegmentebene des anterioren Unterlappensegments zu erkennen sowie eine durchwandernde Embolisationsspirale (๑ Abb.4).

Vor der geplanten endoskopischen Entfernung der Spirale wurden zur Reduktion des Blutungsrisikos die vorgeschalteten Abschnitte der rechten Unterlappenarterie mit Histoacryl/Lipiodol verklebt. Dadurch waren die vorbeschriebenen Gefäßabschnitte komplett von der Durchblutung ausgeschaltet, die AV-Shunts füllten sich ebenfalls nicht mehr ( Abb.5).

Präinterventionell begannen wir eine kalkulierte antibiotische Therapie. Im Intervall erfolgte dann bei normalen Entzündungszeichen komplikationslos die bronchoskopische Entfernung der durchwanderten Embolisationsspirale im Subsegment von B8 rechts mit der Zange. Anschließend zeigten sich die Subsegmente und Subsubsegmente vergleichsweise groß. Eine selektive Bronchografie zeigte den Sub- und Subsubsegmentbronchus geweitet (৫ Abb.6). 


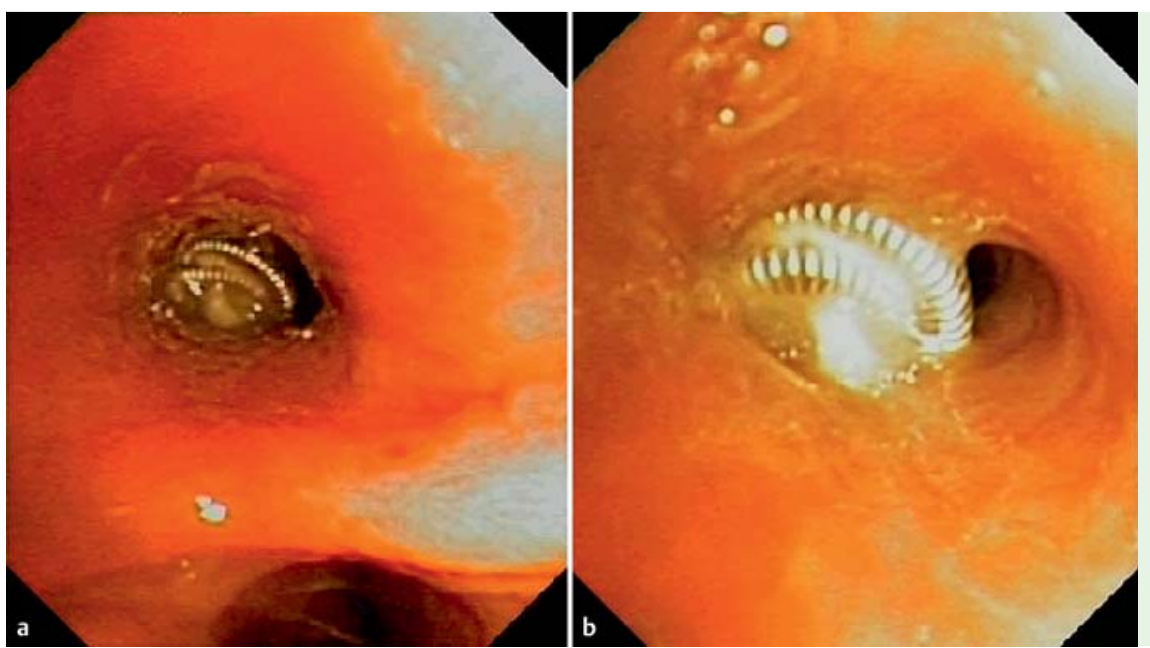

Abb.4 a, b Blick auf durchwandernde Embolisationsspiralen.

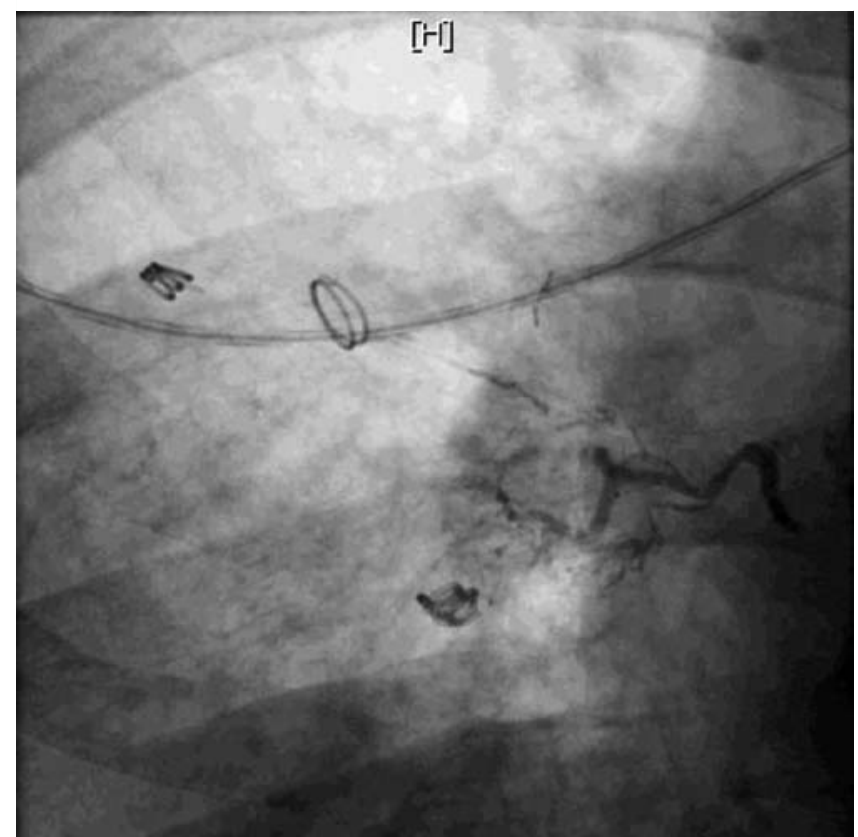

Abb.5 Verklebung Unterlappensegmentarterie rechts.

\section{Diskussion \\ $\nabla$}

Bei Patienten mit HHT und pulmonalen arteriovenösen Malfomationen kann es im Verlauf neben initialen Symptomen wie Dyspnoe oder Zyanose auch zu schwerwiegenden Komplikationen mit Ausbildung einer pulmonalen Hypertonie, Hämoptysen oder Hämatothoraces kommen. Es sollte auch auf zerebrale Komplikationen bei HHT-Patienten mit pAVM geachtet werden. In der Literatur finden sich Ischämien und TIA's bei bis zu 30\% der HHTPatienten mit pAVM [7].

Bei bestehender Klinik mit relevantem Shuntvolumen und zur Prävention schwerer Komplikationen wird eine interventionelle Embolisation der Gefäßanomalien favorisiert. Da sich häufig neue AV-Fisteln bilden und mit dieser Technik auch beidseitige Shunts in einer Sitzung behandelt werden können, ist die interventionelle minimal invasive Methode aufgrund der geringen Komplikationsrate den operativen Behandlungen gegenüber zu bevorzugen. Auch größere Malformationen können mittlerweile interventionell behandelt werden. Kong et al. behandelten einen

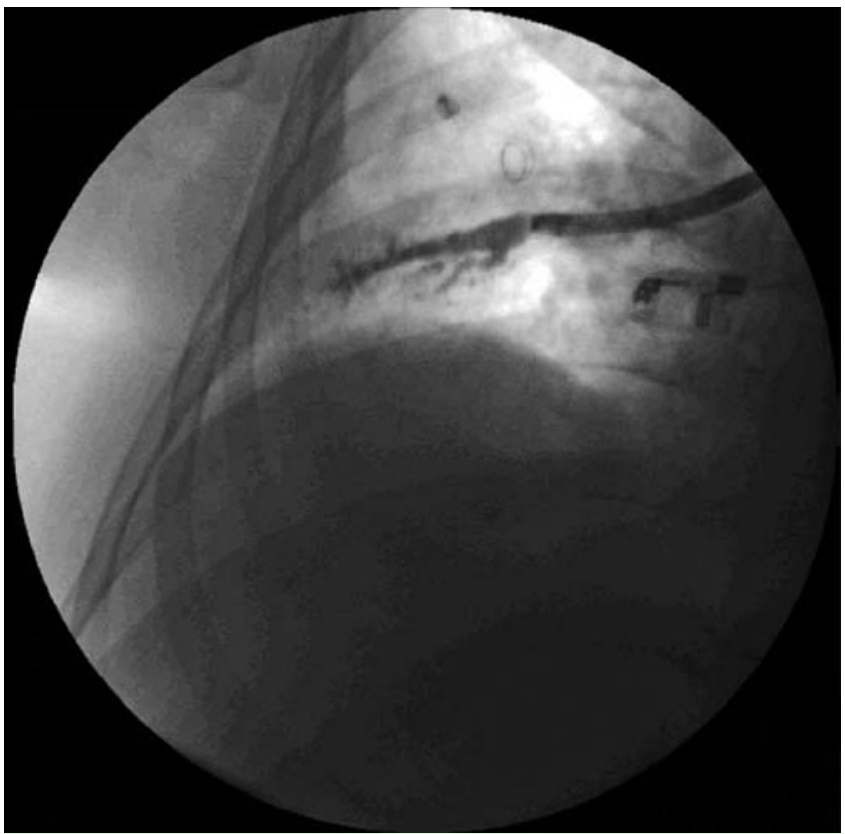

Abb. 6 Selektives Bronchogramm im rechten Unterlappen nach Entfernung der durchwanderten Spiralen.

Patienten mit pulmonaler AV-Fistel und einem Durchmesser von $3 \mathrm{~cm}$ interventionell durch einen Amplatzer Plug [8].

Größere Malformationen können thoraxchirurgisch reseziert werden $[9,10]$.

Im aktuellen Fall zeigten sich die Coils nach Implantation 1997 in der CT-Thorax Kontrolle von 2004 noch regelrecht platziert. Acht Jahre später (2012) sieht man eine Durchwanderung der Embolisationsspiralen sowie abgehustetes Embolisationsmaterial. Bislang ist kein vergleichbarer Kasus veröffentlicht.

Allerdings gibt es Fallberichte bei AV-Malformationen über die Dislokation von Embolisations-Coils von der Niere in das Colon [11], durch die Wand der Blase [12] und des Magens [13].

Ein ähnlicher Fall bei einem Patienten mit Fallot'scher Tetralogie wurde von Wirtz et al. berichtet. Der Patient wurde aufgrund von aortopulmonalen Kollateralen interventionell mit Embolisationsspiralen behandelt. Jahre später stellte er sich mit Hämoptysen vor. Die weitere Umfelddiagnostik zeigte eine dislozierte Embolisationsspirale im rechten Lungenoberlappen mit Anschluss an das Bronchialsystem [14]. 
In Bezug auf unser Fallbespiel ist anzumerken, dass die Versorgung mit 0,35 pushable coils erfolgte. Zu diesem Zeitpunkt war eine dichte Packung der Coils aufgrund der Materialrigidität der Coils nicht möglich. Bei Verwendung moderner, flexibler fibered-coils wird eine bessere Verankerung erreicht, das Risiko einer Coil-Dislokation ist somit deutlich geringer.

\section{Schlussfolgerung}

Bei Hämoptysen nach Interventionen mit Embolisationsspiralen sollte auch Jahre nach deren Implantation neben dem Verdacht auf neue AV-Malformationen an durchwanderte Spiralen gedacht und eine entsprechende Diagnostik mittels Thorax-Kontrastmittel-CT und Bronchoskopie durchgeführt werden.

\section{Interessenkonflikt}

$\nabla$

Die Autoren geben an, dass kein Interessenkonflikt besteht.

\section{Literatur}

1 Sauners WH. Epistaxis. Minn Med 1967; 50: 849-854

2 Shovlin CL, Letarte M. Hereditary hemorrhagic telangiectasia and pulmonary arteriovenous malformations: issues in clinical management and review of pathogenetic mechanisms. Thorax 1999; 54: 714-729

3 Maher CO, Piepgras DG, Brown RD et al. Cerebrovascular manifestions in 321 cases of hereditary hemorrhagic telangiectasia. Stroke 2001; 32: $877-822$

4 Cottin V, Chinet T, Lavolé A et al. Pulmonary arteriovenous malformations in hereditary hemorrhagic telangiectasia: a series of 126 patients. Medicine (Baltimore) 2007; 86: 1-17

5 Haitjema T, Westermann CJ, Overtoom TT et al. Hereditary hemorrhagic telangiectasia (Osler-Weber-Rendu disease): new insights in pathogenesis, complications, and treatment. Arch Intern Med 1996; 156: 714 719

6 Burke CM, Raffin TA. Pulmonary arteriouvenous malformations, aneurysms and reflections. Chest 1986; 89: $771-772$

7 Moshagen V, Sahl H, Wessel K. Zerebraler Abszess als Erstmanifestation einer pulmonalen arteriovenösen Malformation bei Morbus Osler. Akt Neurol 2010; 37: 89-91

8 Kong JH, Oh TY, Kim JT et al. Transcatheter Embolization of Giant Pulmonary Arteriovenous Malformation with an Amplatzer Vascular Plug II. Korean J Thorac Cardiovasc Surg 2012; 45: 326-329

9 Georghiou GP, Berman M, Vidne BA et al. Pulmonary arteriovenous malformationtreated by lobectomy. European Journal of Cardio-thoracic Surgery 2003; $24: 328-330$

10 Metin K, Karaçelik M, Yavaçcan $O$ et al. Surgical treatment of pulmonary arteriovenous malformation: report of two cases and review of the literature. J Int Med Res 2005; 33: 467-71

11 Yoon JW, Koo JR, Baik GH et al. Erosion of embolization coils and guidewires from the kidney to the colon: delayed complication from coil and guidewire occlusion of renal arteriovenous malformation. Am J Kidney Dis 2004; 43: 1109-1112

12 Schaeffer EM, Bivalacqua TJ, Hortopan S et al. Cystoscopic management of an extruded coil from arteriovenous fistula embolization using arthroscopic instruments. J Endourol 2008; 22: 1527-1529

13 Dinter DJ, Rexin $M$, Kaehler $G$ et al. Fatal coil migration into the stomach 10 years after endovascular celiac aneurysm repair. J Vasc Interv Radiol 2007; 18: $117-120$

14 Wirtz DJ, Bhatt NY, Roble SL et al. Endobronchial erosion of vascular embolic coil. Am J Respir Crit Care Med 2012; 185: 682 